\author{
Xavier Escaler \\ Center for Industrial Diagnostics (CDIF), \\ Universitat Politècnica de Catalunya (UPC), \\ Avinguda Diagonal 647, \\ 08028 Barcelona, Spain \\ e-mail: escaler@mf.upc.edu \\ Mohamed Farhat \\ Laboratory for Hydraulic Machines \\ (LMH-IMHEF), \\ École Polytechnique Fédérale de Lausanne \\ (EPFL), \\ Avenue de Cour 33bis, \\ CH-1007 Lausanne, Switzerland \\ e-mail: mohamed.farhat@epfl.ch
}

Eduard Egusquiza

Center for Industrial Diagnostics (CDIF),

Universitat Politècnica de Catalunya (UPC),

Avinguda Diagonal 647,

08028 Barcelona, Spain

e-mail: egusquiza@mf.upc.edu

\author{
Francois Avellan \\ Laboratory for Hydraulic Machines \\ (LMH-IMHEF), \\ École Polytechnique Fédérale de Lausanne \\ (EPFL), \\ Avenue de Cour 33bis, \\ CH-1007 Lausanne, Switzerland \\ e-mail: francois.avellan@epfl.ch
}

\title{
Dynamics and Intensity of Erosive Partial Cavitation
}

An experimental work has been carried out to investigate the dynamic behavior and the intensity of erosive partial cavitation on a 2-D hydrofoil. Both sheet (stable) and cloud (unstable) cavitation have been tested in a cavitation tunnel for various free stream velocities. Special attention has been given to validate the use of acceleration transducers for studying the physical process. In particular, the modulation in amplitude of the cavitation induced vibrations in a high frequency band has allowed us to determine the shedding frequency and the relative intensity of the collapse process for each testing condition. Regarding the cavity dynamics, a typical Strouhal value based on its length of about 0.28 has been found for cloud cavitation; meanwhile, for sheet cavitation, it presents a value of about 0.16. Furthermore, the level of the vibration modulation in the band from $45 \mathrm{kHz}$ to $50 \mathrm{kHz}$ for cloud cavitation shows a power law dependency on the free stream velocity as well as a good correlation with the pitting rate measured on stainless steel samples mounted on the hydrofoil. [DOI: 10.1115/1.2742748]

Keywords: partial cavitation, vibration modulation, dynamics, erosion

\section{Introduction}

1.1 Unsteady Attached Cavities. Attached cavitation appears as macrocavities that develop and attach on a solid wall placed in the flow. The various forms of attached cavitation on the suction side of hydrofoils have been extensively investigated experimentally for many years by, for example, Franc and Michel [1] and Avellan et al. [2]. Numerical simulations (Kubota et al. [3]) and theoretical models (Watanabe et al. [4]) have also been developed, although the results have been very limited up to now. In general terms, two types of unsteadiness with distinctive character have been observed based on the attached cavity length $l$ (Leroux et al. [5]). Partial cavitation occurs for $l / c<3 / 4$ and transitional cavitation occurs for $l / c>3 / 4$, where $c$ is the chord length. An equivalent parameter is $\sigma / 2 i$ that has been found to be about 4 in the transition of these two types of cavitation and higher than 4 for partial cavitation by Kjeldsen et al. [6]. The experimental results seem to indicate that for transitional cavitation, the main cavity dynamics would be governed by a shock-wave phenomenon; meanwhile, for partial cavitation, the predominant mechanism is the re-entrant jet (Callenaere et al. [7]).

Partial cavitation on the suction side of a hydrofoil can present two different regimes, depending on the hydrodynamic conditions. One of them, i.e., sheet cavitation, is characterized by a thin stable cavity with a smooth and transparent interface. At the rear part, the cavity closure presents a slight and weak pulsation due to the shedding of small cavitation vortices. The other regime, i.e., cloud cavitation, shows a strong unsteadiness and a pulsating behavior

Contributed by the Fluids Engineering Division of ASME for publication in the JourNal of FluIDS ENGINEERING. Manuscript received October 13, 2006; final manuscript received January 15, 2007. Review conducted by Joseph Katz. that provokes significant oscillations of the cavity length. The cavity interface is wavy and turbulent. Large U-shaped transient cavities and clouds of cavities are shed away downstream of the cavity closure that collapses violently (Avellan and Dupont [8]). Strong pressure pulses are applied on the solid surface due to the shock waves emitted at the final stage of the cavity collapses (Avellan and Farhat [9]). Consequently, cloud cavitation is found to be a very aggressive form of partial cavitation with a high erosive power compared with sheet cavitation that seems to present a lower risk (Quang et al. [10]).

The majority of experimental and numerical studies have concluded that the shedding frequency of partial cavitation follows a Strouhal law based on the main cavity length. For cloud cavitation, the cavity dynamics clearly show a $\mathrm{St}_{l}$ value of about 0.30 (Leroux et al. [11]); nevertheless, there are no analogous results found for sheet cavitation up to now. The low intensity cavity fluctuations observed with sheet cavitation make it difficult to characterize its behavior.

1.2 Erosion in Hydraulic Machines. Cavitation erosion of runner/impeller blades is a common and relevant problem in hydraulic machinery. The experience based on erosion cases observed in the blades of actual water turbines seems to indicate that it is caused by a partial cavity starting next to the leading edge and with a maximum length below $\frac{3}{4}$ of the chord. When damage occurs, it is generally very aggressive and requires continuous inspection of the runner/impeller to prevent the blades from breaking off. Such strong erosive power appears to be related to the unsteady behavior of the attached partial cavity, as in an isolated hydrofoil (Bourdon et al. [12]). Nevertheless, the basic cavitation mechanisms in actual machines are not yet well known. It is believed that there is a strong interaction with the complex flow features induced by the machine dynamic behavior. For instance, 
in turbines erosive cavitation is difficult to predict since it usually occurs only under certain machine operating conditions (Escaler et al. [13]). To improve our understanding of this phenomenon, it would be appropriate to carry out a detailed investigation of the dynamics and intensity of both sheet and cloud cavitation in the laboratory. Thinking in the latter application of these results in actual machines, where there are strong difficulties in installing instrumentation and measuring without significantly affecting the machine operation, it has been decided to base our research on vibration measurements.

1.3 Cavitation Induced Vibrations. General research on erosive cavitation was started with the idea that noise and vibrations could be used as indicators of cavitation erosive power (Kato et al. [14], Simoneau et al. [15], and Bourdon et al. [16]). Given a hydrofoil in a cavitation tunnel, partial cavities shed clouds of bubbles and cavitation vortices in a periodic way synchronized with the main cavity oscillation. The cloud collapse takes place just downstream of the main cavity and the solid wall is impacted by emitted shock waves. Each collapse results in a high pressure, short duration pulse that excites the structure like a hammer hitting in a small area. As a consequence, vibrations with a broadband spectral content reaching very high frequencies (above tenths of $\mathrm{kHz}$ ) are induced on the structure at the end of each shedding period.

In laboratory measurements, dynamic pressure transducers flush mounted in the test section walls or embedded on the hydrofoil suction side can be used to characterize partial cavitation. Test section pressure signals are very useful to detect the pressure pulsations generated by the main cavity oscillation and the noise due to the cavity collapses. However, it is well known that only a given percentage of the collapses are close enough to the surface to provoke erosion. Therefore, these signals are not adequate if we are concerned about cavitation erosion quantification. Embedded hydrofoil wall pressure signals are obtained with small sized transducers that can only measure local phenomena occurring just above them. Usually, their number is small so that they monitor only a small area of the region of interest. Moreover, their use in real machines is difficult and expensive. Consequently, high frequency acceleration transducers mounted on the external parts of the hydrofoil support and as close as possible to it, are an alternative way of investigating the physical process behind erosive partial cavitation on hydrofoils. They are mechanically linked to the hydrofoil structure and they can detect all pulsating forces acting on the hydrofoil wall that are directly related to erosion.

1.4 Analysis of Vibrations. The use of high frequency vibrations for the study of cavitation erosion started with the evaluation of the overall acceleration level (RMS value) in a broadband frequency range. However, this quantifier is also dependent on the level of vibrations induced by the main free cavitation flow. An improvement of this method arose with the observation that the instantaneous vibration levels are lower during the cloud cavitation inception and its development period. Then, during the collapse stage, the levels obviously increase. As a result, the cavitation induced vibrations appear to be modulated in amplitude at the particular frequency of the main cavity dynamics. Moreover, the modulation level should be related to the erosion intensity. By an adequate demodulation technique, these frequencies can be extracted and their amplitude can be estimated. Abbot et al. [17] were able to validate in hydrofoils and in actual water turbines the use of full-wave rectification spectral analysis for this purpose. Nowadays, a similar modulation analysis is applied but based on digital signal processing [13] which gives more flexibility and the possibility to explore more in detail the experimental data. To finish, just indicate that the complete prediction of cavitation erosion is also dependent on the metallurgical characteristics of the material under attack.

1.5 Objectives. The main objective of the current work is to investigate the dynamic and erosive characteristics of both sheet

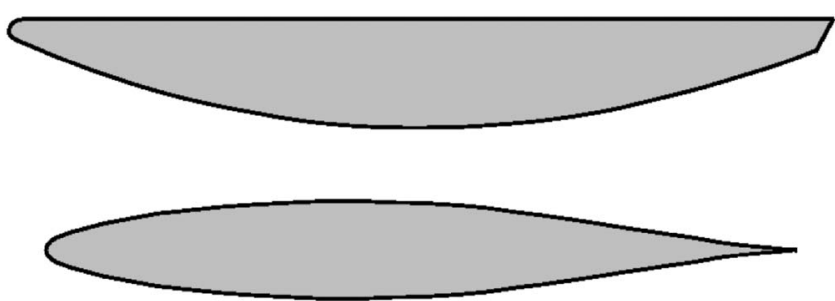

Fig. 1 Cross sections of the plane-convex (top) and of the NACA 65012 (bottom) hydrofoils

and cloud cavitation by the use of cavitation induced vibrations. In particular, it is intended to find out the characteristic shedding frequency and the amplitude of the modulation energy. Considering the latter as an indicator of cavitation erosion intensity, its dependence with the free stream velocity will then be evaluated. It is also intended to find its correlation with the erosion rate measured on the material.

\section{Experimental Setup and Procedure}

2.1 High Speed Cavitation Tunnel. The experiments have been carried out in the Laboratory for Hydraulic Machines-École Polytechnique Fédérale de Lausanne (LMH-EPFL), high-speed cavitation tunnel. This closed loop with circulating water is specially designed for the study of cavitation in hydrofoils [18]. A free stream velocity $\left(U_{\text {inf }}\right)$ up to a maximum of $50 \mathrm{~m} / \mathrm{s}$ can be reached within a rectangular test section of $150 \times 150$ $\times 750 \mathrm{~mm}^{3}$. A revolving bedplate flange provides a rigid mounting base for the hydrofoil to be tested with the possibility of varying its angle of incidence $(i)$. The cavitation number $(\sigma)$, which is continuously controlled, can be adjusted as desired.

2.2 Hydrofoils. Two bi-dimensional hydrofoils with rounded leading edges have been used to generate erosive partial cavitation. One of them is a NACA 65012 and the other one has a plane-convex cross section with a flat suction side and a circular pressure side (see Fig. 1). Their chord lengths are $91.1 \mathrm{~mm}$ and $100 \mathrm{~mm}$, respectively. They have a spanwise length of $150 \mathrm{~mm}$ and a maximum thickness of approximately $12 \mathrm{~mm}$. To carry out erosion tests on materials, both hydrofoils permit us to install replaceable samples on their suction sides.

2.3 Flow Visualization. To identify the regime of the partial cavity and to measure its length, the hydrofoil suction side has been observed through the tunnel transparent windows by means of a digital CCD camera combined with a stroboscopic lamp. Typical photographs of sheet and cloud cavitation are shown in Fig. 2.

2.4 Vibration Measurement. The cavitation induced vibrations can be measured with miniature piezoelectric accelerometers Bruel\&Kjaer type 8309 (BK) and Kistler type 8614A500M1 (K). Their theoretical mounted resonant frequencies are of about $180 \mathrm{kHz}$ and $125 \mathrm{kHz}$, respectively. The BK is bolted, meanwhile, the $\mathrm{K}$ is fixed with wax to the structure. These transducers can be oriented in vertical $(\mathrm{V})$ or horizontal $(\mathrm{H})$ directions inside the revolving bedplate on a special support rigidly attached to the flange (see Fig. 3). The K accelerometers can also be attached on the flange (close) or on the external side of the bedplate (far) in a horizontal direction.

The signals from the transducer are filtered with a Wavetek Butterworth band-pass filter for noise reduction and anti-aliasing prior to being recorded with an analog-to-digital converter LeCroy 6810. The data are then transmitted to the PC acquisition software through a general purpose interface bus (GPIB) to computer automated measurement and control (CAMAC) interface module 


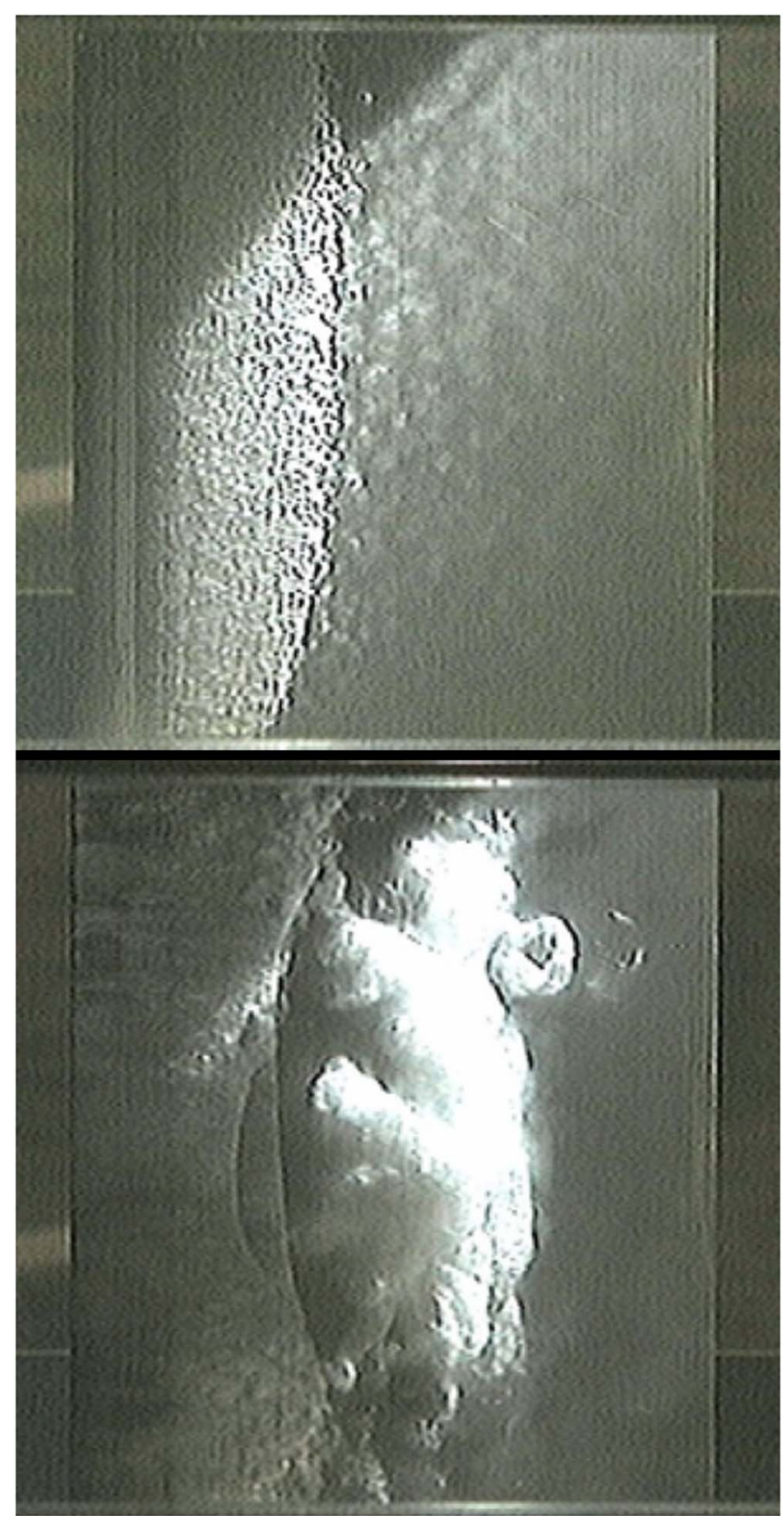

Fig. 2 Partial cavitation on a 2-D hydrofoil. Top: Sheet cavitation at $U_{\text {inf }}=30 \mathrm{~m} / \mathrm{s}, \sigma=0.7$, and $i=2$ deg. Bottom: Cloud cavitation at $U_{\text {inf }}=30 \mathrm{~m} / \mathrm{s}, \sigma=1.2$, and $i=5 \mathrm{deg}$. Flow from left to right.

LeCroy 6010. The low frequency cutoff is usually set at $2 \mathrm{kHz}$. Typical sampling frequencies are $200 \mathrm{kHz}$ and $500 \mathrm{kHz}$.

2.5 Spectral Content and Amplitude Demodulation. The power spectral density (PSD) of the absolute accelerations is calculated from an averaged auto power spectrum using a smoothing Hanning window.

The raw time signals can be band-pass filtered in a given frequency band using an adjustable Butterworth digital filter with ripple 10 , attenuation 40 , and order 2 . The envelope of the filtered time signal is obtained from the analytic signal using the Hilbert transform (see the details of this processing technique in [13]). The frequency content of the envelope shows the main amplitude modulation frequencies.

An example of the cavitation induced vibrations measured with the BK accelerometer in the band from $45 \mathrm{kHz}$ to $50 \mathrm{kHz}$ and the corresponding envelope is shown in Fig. 4. This signal corre-

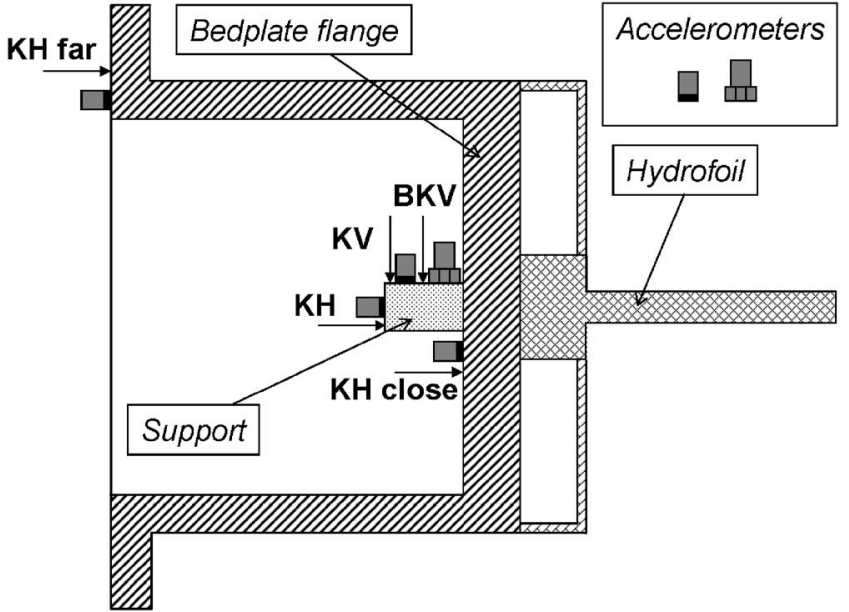

Fig. 3 Section of the hydrofoil, the revolving bedplate, and the support for the accelerometers. Schematic of the transducer locations and orientations.

sponds to unstable cloud cavitation on the NACA hydrofoil tested at $30 \mathrm{~m} / \mathrm{s}$ with $6 \mathrm{deg}$ of incidence and a maximum cavity length of about $40 \%$ of the chord length.

\section{Preliminary Tests}

In order to define the final experimental configuration, a preliminary study of the influence of various measurement and analysis parameters was carried out.

First of all, two types of accelerometers and two orientations were simultaneously tested under cloud cavitation conditions on the plane-convex hydrofoil, in particular, the $\mathrm{BKV}, \mathrm{KV}$, and $\mathrm{KH}$ (refer to Fig. 3). Figure 5 shows the PSD and the amplitude modulation in the band from $45 \mathrm{kHz}$ to $50 \mathrm{kHz}$ of the measured vibrations. The two $\mathrm{K}$ sensors with different orientations measure a similar spectral content but it differs from the BK results. The advantage of this last sensor is that it can detect cavitation excitation up to frequencies of $200 \mathrm{kHz}$. Regarding the amplitude demodulation, this technique appears to work correctly for the same spectral band in the three measurements. A distinctive peak is found for a $\mathrm{St}_{l}$ number of 0.3 which is what is expected for a mean cavity length of $50 \%$ of the chord. In addition, the height of the modulating peak relative to its corresponding baseline is found to be the same for the three sensors. This indicates that, although the absolute level of the amplitude modulation is different, the envelopes are proportional among the sensors. Thus, the relative modulation level is analogous.

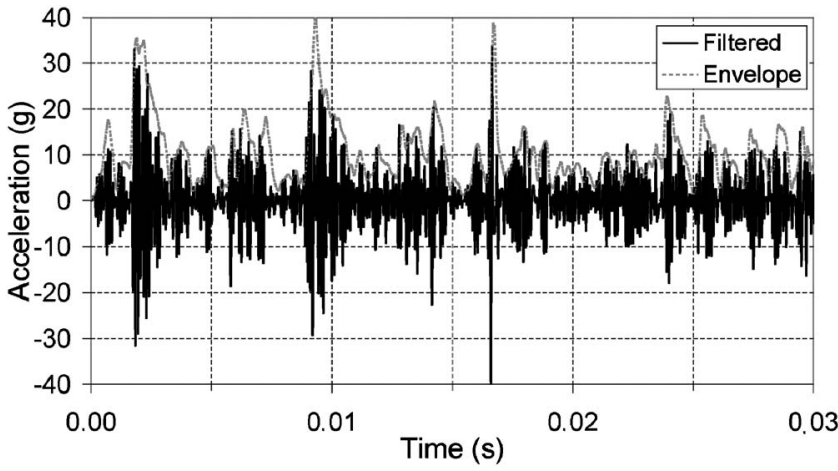

Fig. 4 Filtered time signal and corresponding envelope of cavitation induced vibrations in the band from $45 \mathrm{kHz}$ to $50 \mathrm{kHz}$ $\left(i=6 \mathrm{deg}, U_{\mathrm{inf}}=30 \mathrm{~m} / \mathrm{s}, / / c=0.4\right.$, and $\left.\sigma=1.6\right)$ 

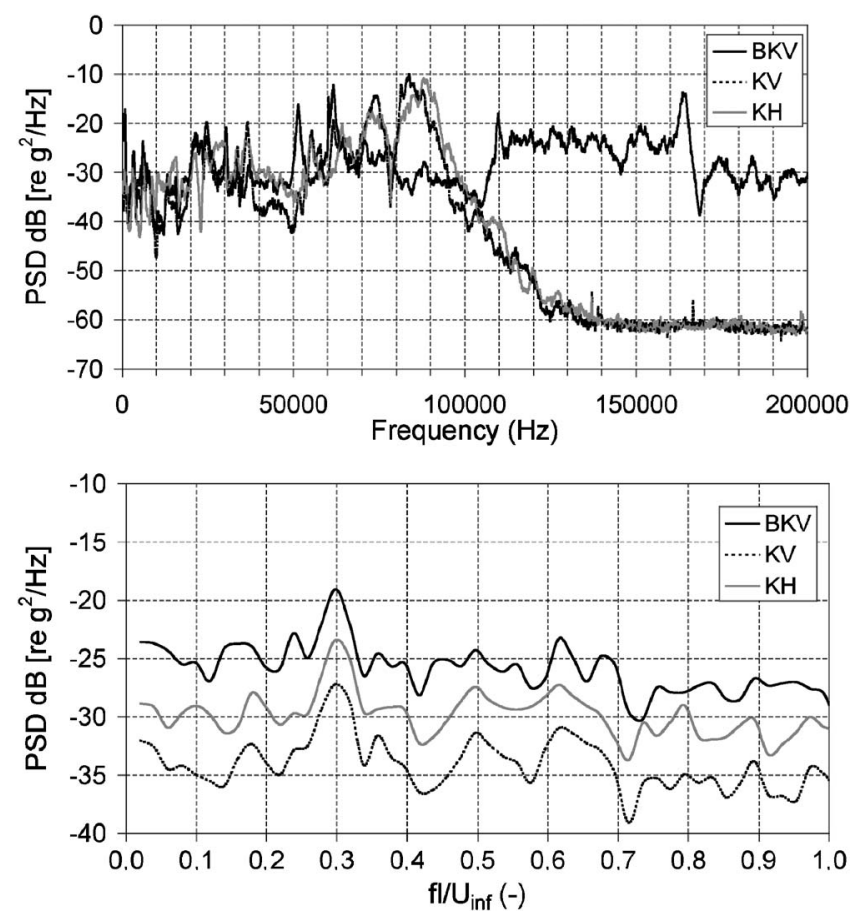

Fig. 5 PSD (top) and modulation in the band from $45 \mathrm{kHz}$ to $50 \mathrm{kHz}$ (bottom) of cloud cavitation vibrations measured on the plane-convex hydrofoil with BKV, KV, and $\mathrm{KH}\left(i=0.8 \mathrm{deg}, \boldsymbol{U}_{\text {inf }}\right.$ $=35 \mathrm{~m} / \mathrm{s}, I / c=0.5$, and $\sigma=0.6$ )

Second, the transmissibility properties of the hydrofoil and its support were also considered by comparing simultaneous measurements with the same type of accelerometer $(\mathrm{K})$ and orientation $(\mathrm{H})$ at two different distances from the source of excitation again with the plane-convex hydrofoil. In Fig. 6, the resulting spectral content and the amplitude modulation are plotted. As in the previous analysis, although the shape of the spectra presents slight differences, the peak of the main amplitude modulation frequency has about the same height within the two measurements. This indicates that in this particular setup, the signal is linearly and uniformly attenuated between the two measuring locations. Thus, the measured structural response to the hydraulic excitation can be considered as a valid indicator of the excitation characteristics.

Finally, the effect of selecting the frequency limits for the amplitude demodulation was also checked. In Fig. 7, the results obtained by filtering at various bands of $5 \mathrm{kHz}$ width in the range from $5 \mathrm{kHz}$ to $80 \mathrm{kHz}$ are plotted together for a given BKV measurement with the NACA hydrofoil. Any band above $5 \mathrm{kHz}$ is suitable to detect the main modulation frequency. However, only above $15 \mathrm{kHz}$ are the modulation results analogous in terms of amplitude relative to the spectrum baseline, i.e., $\mathrm{PSD}_{b}$.

Therefore, based on these preliminary results, it was decided to measure the vibrations during the final tests with the BK accelerometer in vertical orientation on the bedplate flange support. The frequency band from $45 \mathrm{kHz}$ to $50 \mathrm{kHz}$ was selected for the amplitude demodulation analysis of the vibrations because it showed a significant signal level.

\section{Final Erosion Tests}

4.1 Hydrodynamic Conditions and Erosion Results. The vibration and erosion tests have been carried out at tunnel free stream velocities of $15,20,25$, and $30 \mathrm{~m} / \mathrm{s}$ with the NACA 65012 hydrofoil. The incidence angle has been fixed to $4 \mathrm{deg}$ and $6 \mathrm{deg}$. The sigma has been adjusted to generate cavity lengths of about $20 \%$ and $40 \%$ of the chord length. Visual observation has permit-
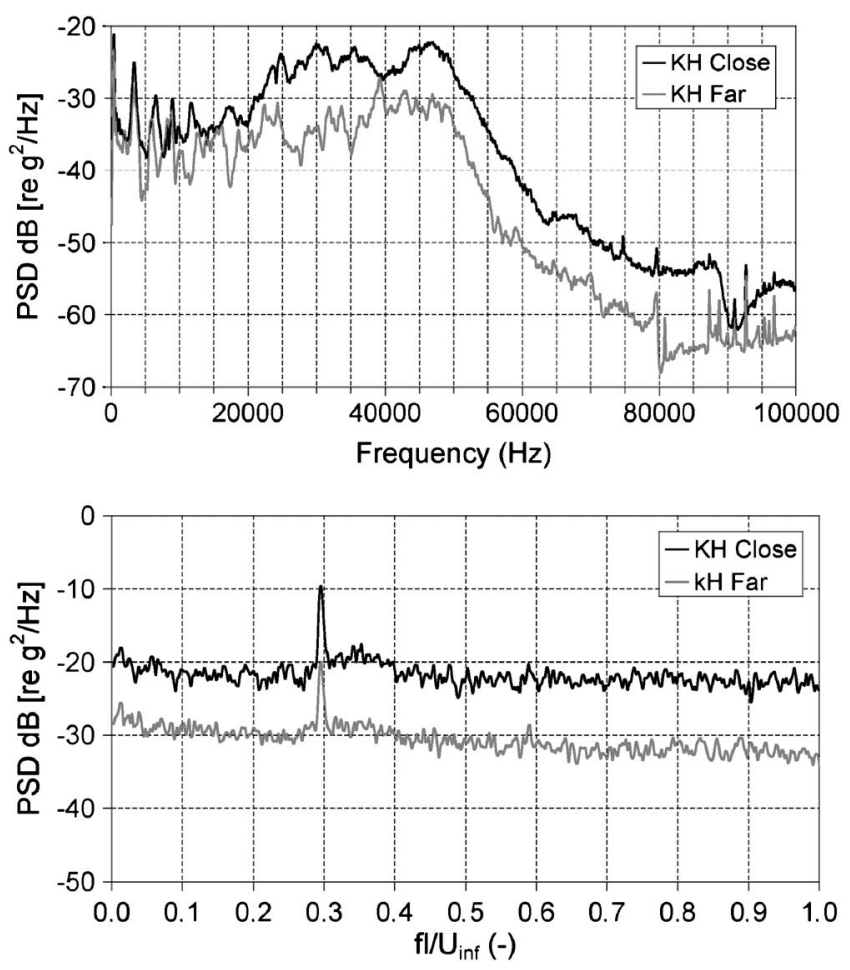

Fig. 6 PSD (top) and modulation in the band from $45 \mathrm{kHz}$ to $50 \mathrm{kHz}$ (bottom) of cloud cavitation vibrations measured on the plane-convex hydrofoil with two $\mathrm{KH}$ at different distances ( $i$ $=5 \mathrm{deg}, U_{\text {inf }}=35 \mathrm{~m} / \mathrm{s}, / / c=0.3$, and $\sigma=1.1$ )
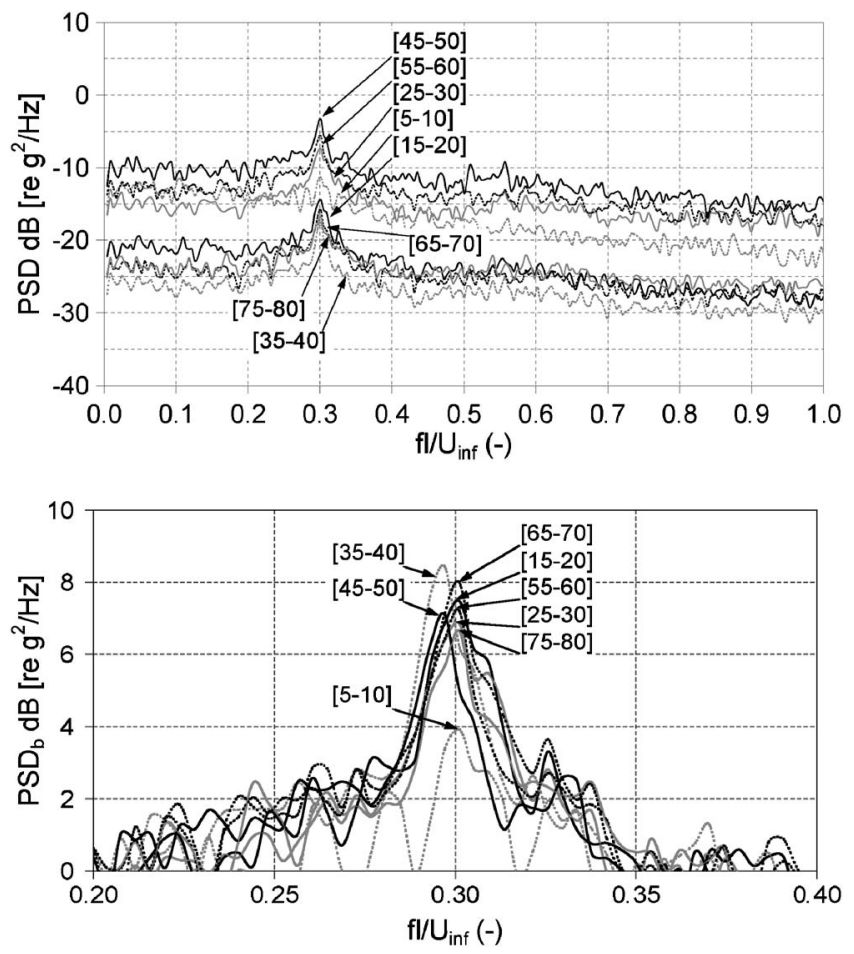

Fig. 7 Modulation results (top) and comparison of their amplitudes relative to the baselines (bottom) for different frequency bands (in $\mathrm{kHz}$ ) of cloud cavitation vibrations measured on the NACA hydrofoil with BKV ( $i=6 \mathrm{deg}, U_{\mathrm{inf}}=30 \mathrm{~m} / \mathrm{s}, / / c=0.4$, and $\sigma=1.6)$ 
Table 1 Testing conditions $\left(i, / / c, U_{\text {inf }}\right)$ and corresponding values of $\sigma, \sigma / 2 i, t, R_{\text {ave }}, \tau, f_{\max }, \mathrm{St}_{l}, \mathrm{RMS}$ value of accelerations and mean square value of $A D_{b}$ in the band from $45 \mathrm{kHz}$ to $50 \mathrm{kHz}$

\begin{tabular}{|c|c|c|c|c|c|c|c|c|c|c|c|}
\hline $\begin{array}{c}i \\
(\operatorname{deg})\end{array}$ & $\begin{array}{l}l / c \\
(\%)\end{array}$ & $\begin{array}{c}U_{\mathrm{inf}} \\
(\mathrm{m} / \mathrm{s})\end{array}$ & $\sigma$ & $\sigma / 2 i$ & $\begin{array}{c}t \\
(\mathrm{~s})\end{array}$ & $\begin{array}{l}R_{\text {ave }} \\
(\mu \mathrm{m})\end{array}$ & $\begin{array}{c}\tau \\
\left(1 / \mathrm{mm}^{2} / \mathrm{s}\right)\end{array}$ & $\begin{array}{l}f_{\max } \\
(\mathrm{Hz})\end{array}$ & $\mathrm{St}_{l}$ & $\begin{array}{l}\text { RMS } \\
(\mathrm{g})\end{array}$ & $\begin{array}{l}A D_{b} \\
\left(\mathrm{~g}^{2}\right)\end{array}$ \\
\hline 4 & 20 & 15 & 1.21 & 8.67 & & & - & - & - & 0.3 & - \\
\hline 4 & 20 & 20 & 1.22 & 8.74 & & & - & - & - & 0.53 & - \\
\hline 4 & 20 & 25 & 1.23 & 8.81 & & & - & 204.47 & 0.16 & 1.39 & 0.014 \\
\hline 4 & 20 & 30 & 1.26 & 9.03 & & & - & 253.30 & 0.17 & 2.75 & 0.248 \\
\hline 4 & 40 & 15 & 1.10 & 7.88 & & & - & - & - & 0.36 & - \\
\hline 4 & 40 & 20 & 1.10 & 7.88 & & & - & 77.82 & 0.16 & 0.8 & 0.002 \\
\hline 4 & 40 & 25 & 1.10 & 7.88 & & & - & 100.71 & 0.16 & 1.75 & 0.026 \\
\hline 4 & 40 & 30 & 1.12 & 8.02 & & & - & 119.02 & 0.16 & 3.42 & 0.166 \\
\hline 6 & 20 & 15 & 1.83 & 8.74 & 7200 & 16.8 & $5.8 \times 10^{-6}$ & 189.21 & 0.25 & 0.85 & 0.015 \\
\hline 6 & 20 & 20 & 1.85 & 8.83 & 2400 & 19.3 & $5.2 \times 10^{-5}$ & 250.24 & 0.25 & 2.59 & 0.160 \\
\hline 6 & 20 & 25 & 1.87 & 8.93 & 480 & 27.0 & $1.5 \times 10^{-4}$ & 387.57 & 0.31 & 5.03 & 0.404 \\
\hline 6 & 20 & 30 & 1.90 & 9.07 & 100 & 27.7 & $4.2 \times 10^{-4}$ & 419.62 & 0.28 & 7.54 & 1.359 \\
\hline 6 & 40 & 15 & 1.55 & 7.40 & 8100 & 17.1 & $6.9 \times 10^{-6}$ & 96.13 & 0.26 & 1.1 & 0.048 \\
\hline 6 & 40 & 20 & 1.58 & 7.55 & 3600 & 34.2 & $1.2 \times 10^{-4}$ & 132.75 & 0.27 & 3.26 & 0.499 \\
\hline 6 & 40 & 25 & 1.60 & 7.64 & 720 & 34.5 & $3.7 \times 10^{-4}$ & 175.48 & 0.28 & 6.16 & 2.002 \\
\hline 6 & 40 & 30 & 1.62 & 7.74 & 150 & 40.8 & $3.5 \times 10^{-3}$ & 225.83 & 0.30 & 10.12 & 7.035 \\
\hline
\end{tabular}

ted us to determine that stable sheet cavitation occurs for $i$ $=4 \mathrm{deg}$ and unstable cloud cavitation for $i=6 \mathrm{deg}$, independently of the rest of the parameters.

Erosion measurements have been carried out by Couty [19] on stainless steel 316L (inox) samples inserted in the NACA hydrofoil. The tests have been carried out during the material incubation period by selecting an adequate exposure time $t$. The surface of the sample has been measured after the test at certain locations along the chord with a 3-D laser profilometer and the pits have been identified and measured with a specific software. Due to the strong resistance of stainless steel, measurable erosion has only been obtained for the most aggressive cavitation conditions corresponding to $i=6 \mathrm{deg}$. The material exposure time, the average pit radius $R_{\text {ave }}$, the average pitting rate $\tau$, and the corresponding hydrodynamic testing conditions are given in Table 1. For additional information, please refer to [16].

4.2 Spectral Analysis. The averaged PSD of the raw cavitation induced vibrations measured with BKV during the tests are presented in Fig. 8. The effect of the free stream velocity on the spectral content is observed on each graph for fixed incidence angles and cavity lengths. Broadly speaking, the increase of $U_{\text {inf }}$ increases the spectrum level uniformly in the entire frequency range from $1 \mathrm{kHz}$ to $100 \mathrm{kHz}$. The spectra appear almost parallel in any range of frequencies.

In Fig. 9, there is a comparison of vibration spectral content at two incidence angles and at two cavity lengths for constant $U_{\text {inf }}$ $=30 \mathrm{~m} / \mathrm{s}$. It is observed that increasing $i$ from $4 \mathrm{deg}$ to $6 \mathrm{deg}$ for the same $l / c$ results in a significant uniform increase of the spectral content in the entire frequency band. For the same $i$, the effect
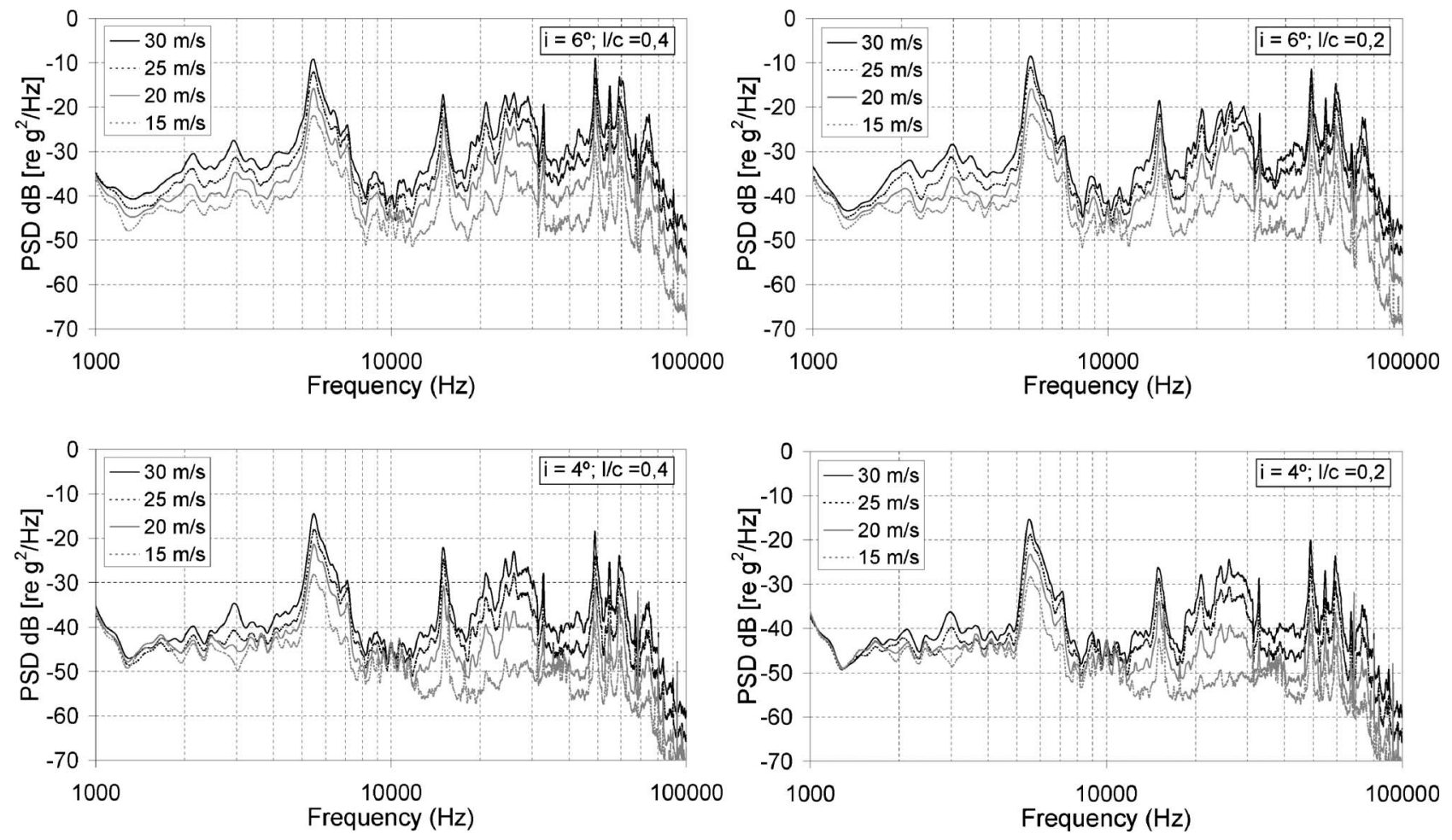

Fig. 8 PSD of the cavitation induced vibrations on the NACA hydrofoil 

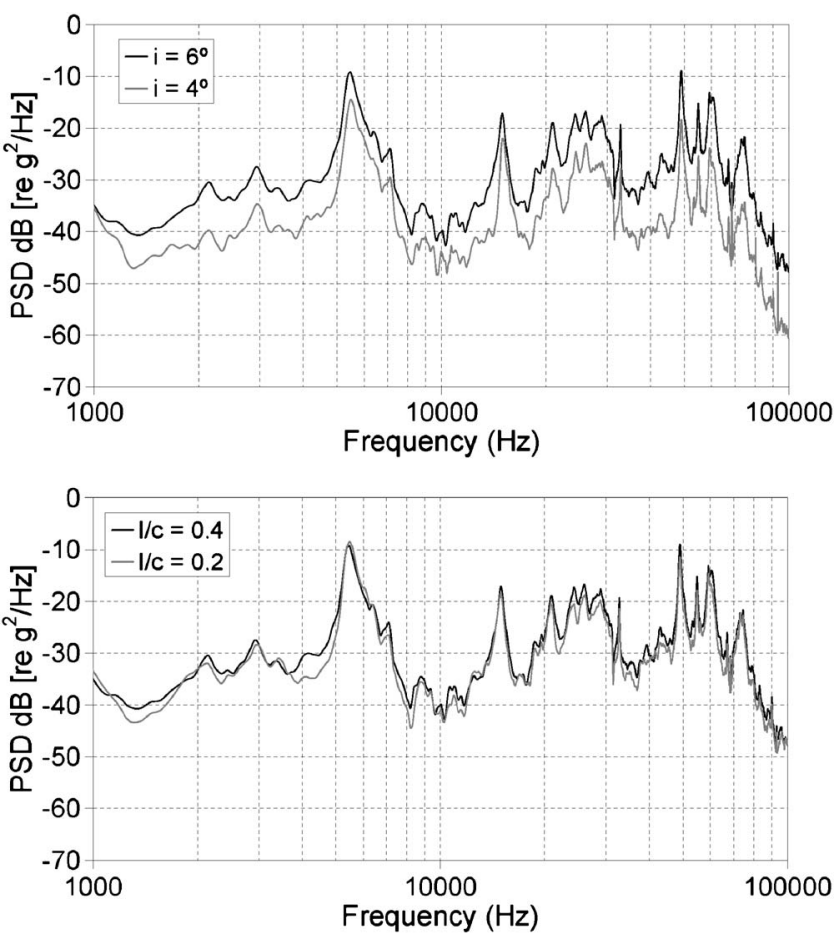

Fig. 9 Comparison of PSD at two incidence angles for the same $/ / c=0.4$ (top) and at two cavity lengths for the same $i$ $=6 \mathrm{deg}$ (bottom) for $U_{\text {inf }}=30 \mathrm{~m} / \mathrm{s}$ on the NACA hydrofoil

of increasing $l / c$ from 0.2 to 0.4 do not show a significant change in the spectra except for a slight parallel increase. In fact, in the first situation, the type of cavitation is changing from sheet to cloud but in the second case, the same type of cavitation is maintained. A similar behavior is observed at any tested $i$ and $U_{\text {inf }}$.

In order to quantify more precisely the energy content of the vibration signals, the RMS values of accelerations in the frequency band from $45 \mathrm{kHz}$ to $50 \mathrm{kHz}$ have been calculated and they are listed in Table 1 . The corresponding overall mean square values have been plotted as a function of $U_{\text {inf }}$ in Fig. 10. These results confirm that the excitation due to cloud cavitation $(i$ $=6 \mathrm{deg})$ is significantly stronger than that of sheet cavitation $(i$ $=4 \mathrm{deg}$ ). Moreover, increasing the cavity length for a given incidence angle from $20 \%$ to $40 \%$ of the chord also enhances the cavitation intensity. For all the types of partial cavitation, the effect of the flow velocity increase has a similar trend with a slope of around 6.4 .

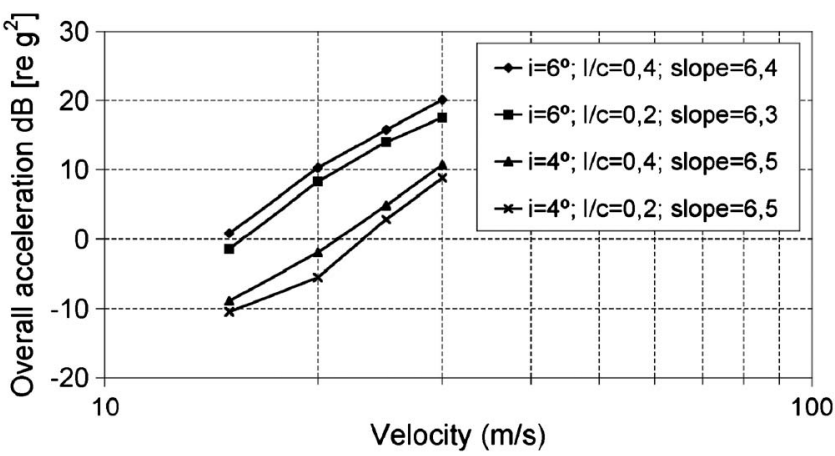

Fig. 10 Overall mean square value of accelerations as a function of $U_{\text {inf }}$ in the band from $45 \mathrm{kHz}$ to $50 \mathrm{kHz}$ on the NACA hydrofoil
4.3 Amplitude Demodulation Analysis. The averaged PSD of the envelopes calculated for the filtered signal in the band from $45 \mathrm{kHz}$ to $50 \mathrm{kHz}$ are plotted in Fig. 11. For the lower free stream velocities at $i=4 \mathrm{deg}$, the amplitude of the vibration acceleration was so low that no reliable demodulation results could be obtained. For the rest of testing conditions, a single amplitude modulation peak has been well identified. Considering the frequency value with maximum amplitude $f_{\max }$, then, the corresponding Strouhal number $\mathrm{St}_{l}$ has been determined. For a more precise evaluation of the modulation energy, the width of the modulation peak and its height relative to the spectrum baseline have been considered. The curve defining the baseline has been calculated with a least-squares fit of the entire spectrum. The range of values around $f_{\max }$ with amplitude levels above the corresponding baseline values have then been considered as part of the modulation peak. And finally, the mean square modulation acceleration of the shedding frequency $A D_{b}$ has been calculated by integrating over this frequency range the PSD values minus the baseline levels. All these results are listed in Table 1.

For all the tests with cloud cavitation corresponding to $i$ $=6 \mathrm{deg}$, the Strouhal number $\mathrm{St}_{l}$ lies in the range from 0.25 to 0.31 with an average value of about 0.275 . However, when sheet cavitation is involved for tests with $i=4 \mathrm{deg}, \mathrm{St}_{l}$ is about 0.16 on average. This is an interesting result that clearly points out a different behavior of the re-entrant jet mechanism for partial cavitation between stable (sheet) and unstable (cloud) regimes.

Another consideration related to the shedding process is observed from the fact that, when the length of the main cavity is reduced from $40 \%$ to $20 \%$ of the chord, the modulation peak is lowered and widened. This would indicate that for a longer cavity, there is a predominating type of collapsing structure with a quite constant shedding frequency; meanwhile, for shorter lengths, the shedding process is not so coherent, and then collapses of less intensity take place with a wider distribution of frequencies.

In Fig. 12, the mean square modulation acceleration $A D_{b}$ is plotted as a function of $U_{\text {inf }}$. The effect of increasing the angle of incidence, the cavity length, and the flow velocity are also well identified. It must be noted that this intensity quantifier is more sensitive to the increase of $U_{\text {inf }}$ than the overall acceleration level in the same band. For high incidence $(i=6 \mathrm{deg})$, the slopes of the linear fits are around 6.7 and for low incidence $(i=4 \mathrm{deg})$, they are even larger, reaching levels of about 10.9.

Finally, the correlation between the $A D_{b}$ level and average pitting rate has been calculated and the result is plotted in Fig. 13 for the tests at $i=6 \mathrm{deg}$. A linear behavior is identified for the cloud cavitation regime with a slope of about 1.1, which appears to agree with the results obtained by Abbot et al. [17] in 1993.

\section{Conclusions}

An experimental investigation on erosive partial cavitation has been carried out based on the measurement of cavitation induced vibrations in a broadband frequency range up to $100 \mathrm{kHz}$.

The use of acceleration transducers mounted on the test section is valid to study the physical characteristics of this type of cavitation.

The analysis of the vibrations' spectral content and the amplitude demodulation of the acceleration signals filtered from $45 \mathrm{kHz}$ to $50 \mathrm{kHz}$ permits us to quantify the cavitation intensity and to identify the main cavity shedding frequency.

A series of tests have been carried out with sheet (stable) and cloud (unstable) cavitation regimes using the NACA 65012 hydrofoil corresponding to incidence angles of $4 \mathrm{deg}$ and $6 \mathrm{deg}$, respectively. In each regime, cavity lengths of about $20 \%$ and $40 \%$ of the chord have been reproduced at various free stream velocities from $15 \mathrm{~m} / \mathrm{s}$ to $30 \mathrm{~m} / \mathrm{s}$.

The shedding process of cloud cavitation follows a constant Strouhal value based on the main cavity length of about 0.28 , meanwhile, for sheet cavitation it follows a constant value of 

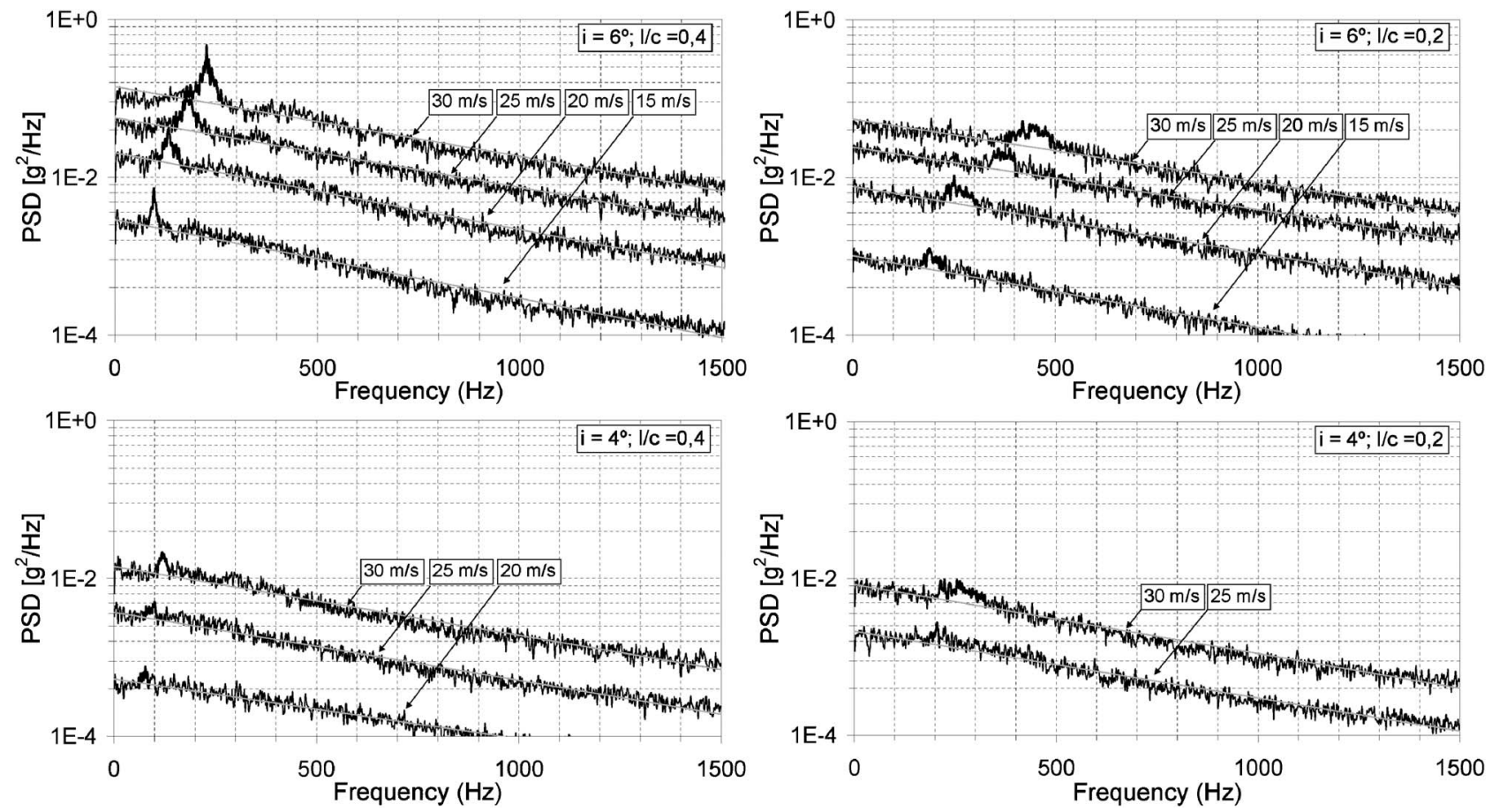

Fig. 11 PSD of vibration modulation spectra in the band from $45 \mathrm{kHz}$ to $50 \mathrm{kHz}$ on the NACA hydrofoil

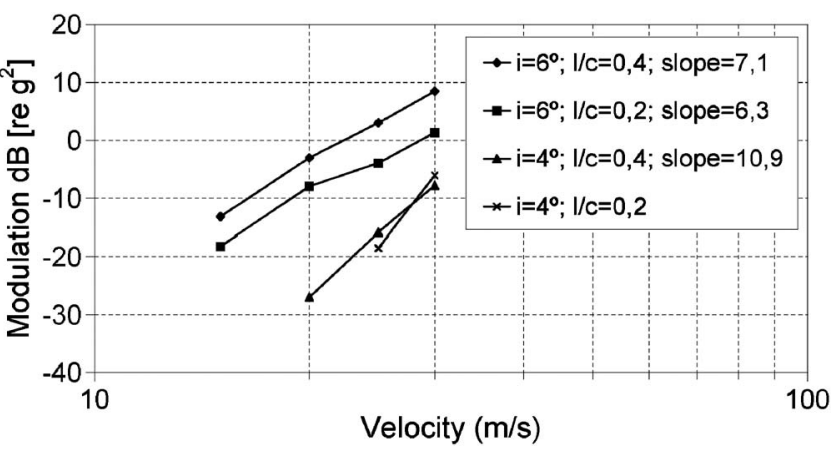

Fig. 12 Mean square level of the vibration modulation as a function of $U_{\text {inf }}$ in the band from $45 \mathrm{kHz}$ to $50 \mathrm{kHz}$ on the NACA hydrofoil

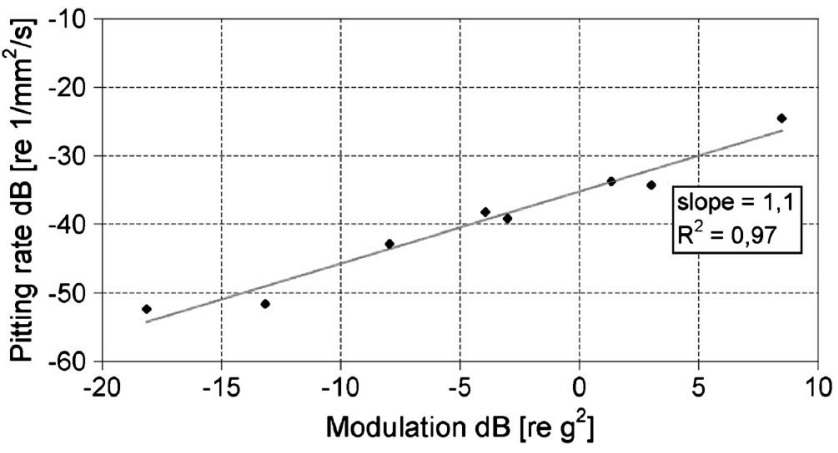

Fig. 13 Average pitting rate on stainless steel as a function of mean square level of the vibration modulation in the band from $45 \mathrm{kHz}$ to $50 \mathrm{kHz}$ at $i=6 \mathrm{deg}$ and linear fit for the NACA hydrofoil about 0.16. At low free stream velocities, no modulation is detected for sheet cavitation. When the length of the main cavity is reduced from $40 \%$ to $20 \%$ of the chord, the modulation frequency peak is lowered and widened out.

The intensity of the erosive partial cavitation has been measured based on the mean square acceleration of the modulation frequency peak. The effect of increasing the flow free stream velocity has been well quantified for cloud cavitation. In particular, the intensity shows a power law dependency on the velocity with an exponent of about 6.7 .

A good correlation has also been found between the modulation level and the actual average pitting rate measured on the hydrofoil stainless steel samples for cloud cavitation.

\section{Acknowledgment}

The members and technical staff of the LMH-EPFL Cavitation Group are thanked for their support and collaboration.

\section{Nomenclature}

$A D_{b}=$ mean square modulation acceleration, relative to baseline $\left(\mathrm{g}^{2}\right)$

$\mathrm{BK}=$ accelerometer Bruel\&Kjaer 8309

$c=$ hydrofoil chord length $(\mathrm{m})$

$f=$ frequency $(\mathrm{Hz})$

$f_{\max }=$ frequency with maximum amplitude $(\mathrm{Hz})$

$\mathrm{H}=$ horizontal orientation

$i=$ incidence angle (deg)

$\mathrm{K}=$ accelerometer Kistler 8614A500M1

$l=$ attached cavity length $(\mathrm{m})$

$p_{\text {inf }}=$ free stream pressure $(\mathrm{Pa})$

$p_{\text {ref }}=$ reference pressure $(\mathrm{Pa})$

$p_{v}=$ vapor pressure $(\mathrm{Pa})$

PSD $=$ power spectral density $\left(\mathrm{g}^{2} / \mathrm{Hz}\right)$

$\mathrm{PSD}_{b}=$ PSD relative to baseline $\left(\mathrm{g}^{2} / \mathrm{Hz}\right)$

$R_{\text {ave }}=$ average pit radius $(\mu \mathrm{m})$

RMS $=$ root mean square

$\mathrm{St}_{l}=$ Strouhal number, $\mathrm{St}_{l}=f l / U_{\mathrm{inf}}$

$t=$ exposure time $(\mathrm{s})$ 


$$
\begin{aligned}
U_{\text {inf }} & =\text { free stream velocity }(\mathrm{m} / \mathrm{s}) \\
\mathrm{V} & =\text { vertical orientation }
\end{aligned}
$$

\section{Greek Letter}

$$
\begin{aligned}
\rho & =\text { density }\left(\mathrm{Kg} / \mathrm{m}^{3}\right) \\
\sigma & =\text { cavitation number, } \sigma=2\left(p_{\mathrm{ref}}-p_{v}\right) /\left(\rho U_{\mathrm{inf}}^{2}\right) \\
\tau & =\text { average pitting rate }\left(1 / \mathrm{mm}^{2} / \mathrm{s}\right)
\end{aligned}
$$

\section{References}

[1] Franc, J. P., and Michel, J. M., 1985, "Attached Cavitation and the Boundary Layer: Experimental Investigation and Numerical Treatment," J. Fluid Mech., 154, pp. 63-90.

2] Avellan, F., Dupont, P., and Ryhming, I., 1988, "Generation Mechanism and Dynamics of Cavitation Vortices Downstream of a Fixed Leading Edge Cavity," Proceedings of the 17th Symposium on Naval Hydrodynamics, The Hague, The Netherlands, Session V, pp. 1-13.

[3] Kubota, A., Kato, H., and Yamaguchi, H., 1992, "A New Modelling of Cavitating Flows: A Numerical Study of Unsteady Cavitation on a Hydrofoil Section," J. Fluid Mech., 240, pp. 59-96.

4] Watanabe, S., Tsujimoto, Y., and Furukawa, A., 2001, "Theoretical Analysis of Transitional and Partial Cavity Instabilities," ASME J. Fluids Eng., 123(3), pp. 692-697.

[5] Leroux, J.-B., Astolfi, J. A., and Billard, J. Y., 2004, "An Experimental Study of Unsteady Partial Cavitation,” ASME J. Fluids Eng. 126(1), pp. 94-101.

[6] Kjeldsen, M., Arndt, R. E. A., and Effertz, M., 2000, "Spectral Characteristics of Sheet/Cloud Cavitation," ASME J. Fluids Eng., 122(3), pp. 484-487.

[7] Callenaere, M., Franc, J. P., Michel, J. M., and Riondet, M., 2001, "The Cavitation Instability Induced by the Development of a Re-Entrant Jet," J. Fluid Mech., 444, pp. 223-256.

[8] Avellan, F., and Dupont, P., 1988, "Cavitation Erosion of the Hydraulic Machines: Generation and Dynamics of Erosive Cavities," Proceedings of the 14th IAHR Symposium, Trondheim, Norway, pp. 725-738.

[9] Avellan, F., and Farhat, M., 1989, "Shock Pressure Generated by Cavitation Vortex Collapse," Proceedings of the 3rd International Symposium on Cavitation Noise and Erosion in Fluid Systems, ASME Winter Annual Meeting, San Francisco, Vol. 88, pp. 119-125.
[10] Quang, L., Franc, J. P., and Michel, J. M., 1989, "Pressure Pulse Distribution Around Cavity Closure," Proceedings of the 3rd International Symposium on Cavitation Noise and Erosion in Fluid Systems, ASME Winter Annual Meeting, San Francisco, Vol. 88, pp. 111-118.

[11] Leroux, J.-B., Coutier-Delgosha, O., and Astolfi, J. A., 2005, “A Joint Experimental and Numerical Study of Mechanisms Associated to Instability of Partial Cavitation on Two-Dimensional Hydrofoil," Phys. Fluids, 17, p. 052101.

[12] Bourdon, P., Simoneau, R., and Avellan, F., 1993, "Erosion Vibratory Fingerprint of Leading Edge Cavitation of a NACA Profile and of a Francis Model and Prototype Hydroturbine," Proceedings of Bubble Noise and Cavitation Erosion in Fluid Systems, ASME Winter Annual Meeting, New Orleans, Vol. 176, pp. 51-67.

[13] Escaler, X., Egusquiza, E., Farhat, M., and Avellan, F., 2006, "Detection of Cavitation in Hydraulic Turbines," Mech. Syst. Signal Process., 20, pp. 983 1007

[14] Kato, H., Ye, Y. P., and Maeda, M., 1989, "Cavitation Erosion and Noise Study on a Foil Section," Proceedings of the 3rd International Symposium on Cavitation Noise and Erosion in Fluid Systems, ASME Winter Annual Meeting, San Francisco, Vol. 88, pp. 41-46.

[15] Simoneau, R, Avellan, F, and Kuhn de Chizelle, Y, 1989 "On Line Measurement of Cavitation Erosion Rate on a 2D NACA Profile," Proceedings of the 3 rd International Symposium on Cavitation Noise and Erosion in Fluid Systems, ASME Winter Annual Meeting, San Francisco, FED-Vol. 88, pp. 95102.

[16] Bourdon, P., Simoneau, R., Avellan, F., and Farhat, M., 1990, "Vibratory Characteristics of Erosive Cavitation Vortices Downstream of a Fixed Leading Edge Cavity," Proceedings of the 15th IAHR Symposium on Modern Technology in Hydraulic Energy Production, Belgrade, Serbia, Vol. 1, Session H(3), pp. 1-12.

[17] Abbot, P. A., Arndt, R. E. A., and Shanahan, T. B., 1993, "Modulation Noise Analyses of Cavitating Hydrofoils," Proceedings of Bubble Noise and Cavitation Erosion in Fluid Systems, ASME Winter Annual Meeting, New Orleans, Vol. 176, pp. 83-94.

[18] Avellan, F., Henry, P., and Rhyming, I., 1987, "A New High Speed Cavitation Tunnel for Cavitation Studies in Hydraulic Machinery," Proceedings of International Symposium on Cavitation Research Facilities and Techniques, ASME Winter Annual Meeting, Boston, Vol. 57, pp. 49-60.

[19] Couty, P., 2001, "Physical Investigation of Cavitation Vortex Collapse," 2463, École Polytechnique Fédérale de Lausanne, Lausanne. 